\title{
The Effects of the COVID-19 Pandemic and Vaccine Knowledge on Vaccine Hesitancy in Adolescents
}

\author{
Claire English ${ }^{1}$ and Lori Cohen ${ }^{1 \#}$ \\ ${ }^{1}$ Mentor High School, Mentor, OH, USA \\ \#Advisor
}

\section{ABSTRACT}

This research study addresses the issue of vaccine hesitancy in adolescents. The research questions of this study include: To what extent does an adolescent's experience with COVID-19 influence their vaccine hesitancy, if an adolescent routinely gets influenza vaccinations will they have a lower vaccine hesitancy, will an individual's vaccine hesitancy impact their willingness to receive a vaccination for COVID-19, and to what extent does an individual's knowledge of vaccines impact their vaccine hesitancy? To obtain participants for this study a random sample of students enrolled in science classes at the researcher's school were selected. The students completed a 4-part survey which included sample demographics, COVID-19 experience, and routine flu vaccinations questions; knowledge of vaccines questions; the Vaccine Hesitancy Scale; and questions regarding the refusal of vaccines. The study cannot conclude that individuals who know someone who has had COVID or know someone who has died of COVID will be less vaccine hesitant. However, the study is able to conclude with a $95 \%$ confidence that individuals who receive the flu vaccine routinely will be less vaccine hesitant. Moreover, it is concluded with greater than $99 \%$ confidence that an individual who is more knowledgeable about vaccines or willing to receive a vaccination for COVID-19 will be less vaccine hesitant. The issue of vaccine hesitancy is not only important to address because of the effectiveness of vaccinations, but it is also critical to reduce vaccine hesitancy during the current pandemic, where vaccinations could stop the spread of COVID-19 and ultimately save lives.

\section{Introduction}

Vaccine hesitancy is a growing concern in the world climate today. In fact, according to the peer-reviewed article "Vaccine hesitancy' among university students in Italy during the COVID-19 pandemic" (2020), the World Health Organization states vaccine hesitancy is "one of the top-ten threats to global health." In addition to this, the article "Parental hesitancy about routine childhood and influenza vaccinations: A national survey" (2020) explains that there is not a plentiful amount of research which details the hesitancy among parents in the U.S. Moreover, the hesitancy among parents is more commonly measured than the vaccine hesitancy of their children, so it can also be said that there is very little information regarding the vaccine hesitancy of adolescents. Vaccines are incredibly important to global health. According to the article "The Anti-vaccination Movement: A Regression in Modern Medicine" (2018) vaccines have led to a great decrease in childhood diseases and have also eradicated certain diseases including smallpox and rinderpest. They have also "nearly eradicated malaria and polio." Emphasizing the effectiveness of vaccines in dropping the rates of diseases, the article "Six common misconceptions about immunization" published by the World Health Organization explains that Hib disease in children has decreased from about 20,000 cases a year in 1990 to 1,419 cases in 1993 in the United States. This cannot be attributed to factors such as sanitation because they did not drastically change between 1990 and 1993, while during these years a vaccine was introduced indicating that it was the cause of the extreme change. Due to the fact that vaccines are incredibly effective in decreasing the rate of disease, gathering more information regarding the population's vaccine hesitancy is crucial because it is necessary to show what steps need to be taken to solve the problem, and whether or not these steps are effective. 
The issue of vaccine hesitancy is incredibly long-standing. The first vaccination was created in Europe in the 18th Century to protect against smallpox, and even then, groups of individuals were resistant to vaccines. In England, Reverend Massey declared that vaccines attempted "to oppose God's punishments upon man for his sins" and that they were "diabolical operations" (Hussain, et. al., 2018). This strong opposition to vaccines led to a social movement against the scientific advancement. The anti-vaccination movement focuses on the belief that vaccines cause more harm to children than good (Hussain, et. al., 2018). Further fueling the fight against these individuals is the rise of misinformation. In the age of technology people are able to find answers to whatever questions they may have, and while this may be generally beneficial, it has also caused the spread of misinformation which can be incredibly dangerous. In relation to vaccines, a study found that $32 \%$ of YouTube videos regarding vaccinations were in opposition, and that these videos had more views and better ratings than those in support of immunizations (Hussain, et al., 2018). This plethora of misinformation encourages those who believe in the anti-vaccination movement. To exhibit this, an article published by Andrew Wakefield, a former British doctor and researcher, claimed that there was a correlation between the measles, mumps, and rubella (MMR) vaccine and development of autism. This article has since been debunked, but the effects are lasting. In the United Kingdom, the rate of the MMR vaccine dropped from 92\% in 1996 to $84 \%$ in 2002. In the United States, the rate of this vaccine declined 2\% (Hussain, et al., 2018). The article "Target the fence-sitters" by Julie Leask (2011) provides an additional example of misinformation leading to lower immunization rates in explaining the attempt to link the Diphtheria-Tetanus-Pertussis (DTP) vaccine with Sudden Infant Death Syndrome (SIDS) in the 1970s. This increase in available information not only has charged the anti-vaccination movement, but it has also, in turn, increased vaccine hesitancy.

Not only is the spread of misinformation incredibly dangerous due to the adverse effects it has on an individual's knowledge of vaccinations, but also an individual's confirmation bias. The article "Confirmation bias" by Iqra Noor (2020) explains the phenomenon as a tendency that people have which makes them "favor information that confirms their existing beliefs or hypotheses." Confirmation bias can occur in several different forms which includes biased searches for information, biased interpretations, or biased memory. Through these various types of confirmation bias people may search for information or choose to understand information a certain way to assert their beliefs about a topic. People may also choose or subconsciously remember information selectively to support their opinions (Noor, 2020). In learning to be aware of confirmation bias and the effects it has on the views of individuals, people can make conscious decisions to be more knowledgeable about important topics. Especially considering the fallacies that exist regarding vaccinations, people must fight against confirmation bias to find reliable information that allows them to gain a greater understanding about the topic.

While the anti-vaccination movement and vaccine hesitancy are closely related, they are not exactly the same. As exemplified in the article "The VACCINES Act: Deciphering Vaccine Hesitancy in the Time of COVID-19" by John McAteer, Inci Yildirim, and Ann Chahroudi (2020), the anti-vaccination movement generally describes individuals who are skeptical of vaccines, while vaccine hesitancy describes a general uncertainty. While there is a greatly established history regarding these topics, the definition of vaccine hesitancy has still been greatly debated and is commonly inconsistent (Kempe et. al., 2020). This furthers the already prominent issue because it creates more difficulty to measure vaccine hesitancy and develop a solution. Specifically, certain definitions of this term may focus on "beliefs about perceived safety, effectiveness, or necessity of vaccines," while other definitions also include the problem of convenience or barriers which prevent vaccinations (Kempe et. al., 2020). The peer-reviewed article "The vaccine hesitancy scale: Psychometric properties and validation" from Vaccine by Gilla K. Shapiro et al. (2017) defines vaccine hesitancy as "the delay in acceptance or refusal of vaccination despite the availability of services." While this is just a simplistic description of vaccine hesitancy, it at least offers a consistent definition allowing for a more feasible comparison.

The problem of vaccine hesitancy is of great importance and has become a global priority. To exemplify this, the Global Vaccine Action Plan was created which aimed to raise vaccination rates in the decade of 2011 to 2020. Furthermore, a working group to research vaccine hesitancy was created by the World Health Organization Strategic Advisory Group of Experts on Immunization, thus exemplifying that there is great global action being taken against 
the growing resistance to vaccines (Shapiro et al., 2017). This global action is likely met with several challenges as there are a plethora of causes for increased vaccine hesitancy. The article, "Mistrust in biomedical research and vaccine hesitancy: the forefront challenge in the battle against COVID-19 in Italy" by Lorenzo Palamenghi, Serena Barello, Stefania Boccia, and Guendalina Graffigna (2020) explains that certain causes of hesitancy in individuals towards the use of vaccinations include "socio-demographic and psychological factors, such as public trust (or mistrust) towards health-care professionals and health authorities." In addition to this, the individual's trust in science also plays a role in their perspective towards vaccinations (Palamenghi et al., 2020). Organizations could potentially be able to see the problem more clearly if the main factors which contribute to vaccine hesitancy were understood. With this greater understanding, a possible solution could possibly be reached.

The issue of vaccine hesitancy leads to great public health dangers as it results in more people resistant towards vaccinating themselves. As a result of this distrust leading to greater rates of resistance towards vaccines, there have been recent outbreaks of diseases including measles, mumps, and diphtheria (Shapiro et al., 2017). This emphasizes how ignoring the importance or effectiveness of vaccinations can lead to deleterious consequences. To exemplify, the article, "Vaccine hesitancy and anti-vaccination movements" by David Isaacs (2019) explains that there have been several measles outbreaks globally in recent years. These have occurred in Venezuela, Ukraine, Yemen, and even the United States. In 2019, there were 1,282 cases of the measles, which is the highest this number has been since 1992 (McAteer, et al., 2020). Higher vaccine hesitancy results in a lower vaccination rate which leads to lower herd immunity in the population. This has the potential to create outbreaks of diseases that were once largely eradicated (Shapiro et al., 2017). Not only is this extremely dangerous for those who have a compromised health, but it also has the potential to increase vaccine hesitancy because it may lead people to believe that vaccines are not effective. However, in truth, the reason these outbreaks are occurring is because not enough people are getting the vaccinations.

In order for a comparison of vaccine hesitancy to be possible, a critical first step is to quantify the level of vaccine hesitancy. This will help preventative steps be taken to solve the problem and also measure the effectiveness of these efforts. Certain measures have already been taken to attempt to examine vaccine hesitancy. The article "The vaccine hesitancy scale: Psychometric properties and validation" introduces several scales such as the eight-item Vaccine Confidence Scale, the 18-item Parental Attitudes about Childhood Vaccines, the 7-item Vaccine Conspiracy Belief Scale, the 9-item Knowledge of Vaccination Scale, the 12-item Vaccination Attitudes Examination Scale, and the 5-item Vaccine Attitude Scale. While it is beneficial to create these scales in order to measure vaccine hesitancy, having so many different types of measurements makes it difficult to consistently compare and analyze data. However, a relatively newer scale called the Vaccine Hesitancy Scale "has been psychometrically validated and encompasses 10 items with Likert responses, including dimensions of vaccine confidence and vaccine risks" (Kempe et al., 2020). Additionally, the effectiveness of this tool has been confirmed through two different studies. To create a more unified approach in addressing the growing issue of vaccine hesitancy, an effective scale must be determined for a collective understanding of the topic.

In addition to the general implications which may include outbreaks of largely eradicated diseases, vaccine hesitancy is also of immense concern in the time of the coronavirus (COVID-19). This disease originated in December of 2019 in Wuhan, China. Additionally, researchers around the globe are working tirelessly to find a vaccine to the virus (McAteer, 2020). The article "Influenza immunization and COVID-19" by Helena C. Maltezou, Kalliopi Theodoridou, and Gregory Poland (2020) explains that this respiratory illness, causing a global pandemic, has killed over 600,000 people as of July 23, 2020 and infected over fifteen million. These drastic numbers are incredibly daunting. Moreover, due to the increased stress on healthcare systems, access to immunizations has greatly decreased in several countries (Maltezo et al., 2020). This indicates that the number of people being immunized is far too low given that resistance towards vaccines has increased and access to immunizations has decreased.

Throughout the pandemic COVID-19 and its effects have been constantly compared to influenza (flu). However, in comparison to the flu, the resulting deaths of COVID-19 are 9.5 to 44.1 times greater than in the peak week of the past seven influenza seasons (Maltezo et al., 2020). This makes it clearly evident that COVID-19 is of great 
seriousness and should not be dismissed as something that will go away on its own. Additionally, provided that vaccine hesitancy is a growing issue, this common comparison may deter people from getting a COVID-19 vaccine, once one is available. To exemplify this, in the 2018 to 2019 influenza season the rate of children from 6 months to 18 years of age who received the flu vaccine was a meager 57.9\% (Kempe et al., 2020). This shows that there is not a great number of people who receive the flu vaccine yearly, and if people associate influenza with COVID-19 then not enough people will get a vaccine. In the article, "High Contagiousness and Rapid Spread of Severe Acute Respiratory Syndrome Coronavirus 2" by Steven Sanche, et. al (2020), the results of the study explains that in order to "effectively stop the spreading of the disease" and gain herd immunity, between $55 \%$ and $82 \%$ of the population must be immune to the virus. This may be achieved through exposure to the virus or by vaccination. This exhibits that the proportion of people who will likely get a vaccine, based on those who receive an influenza vaccination, is not a high enough rate to be confident that herd immunity will be achieved. In addition to this, the demand on healthcare systems due to COVID19 is predicted to be much greater than during a flu season due to the ease of human-to-human transmission of COVID19, large susceptibility in the population, and a higher case fatality rate of COVID-19 (Maltezo et al., 2020). Healthcare facilities are under extreme pressure during this pandemic; a pressure that is much greater than the peak of flu season. With these great pressures, it is important to understand the severity of COVID-19 and the incredible crisis the world is facing. In failing to do this, the vaccine hesitancy among the population will rise, thus decreasing the number of people willing to be vaccinated.

In order to assess the connections of these various factors and their effects on vaccine hesitancy and intentions to vaccinate, this research paper will explore the following questions:

$>$ To what extent does an adolescent's experience with COVID-19 influence their vaccine hesitancy?

$>$ If an adolescent routinely gets influenza vaccinations will they have a lower vaccine hesitancy?

$>$ Will an individual's vaccine hesitancy impact their willingness to receive a vaccination for COVID-19?

$>$ To what extent does an individual's knowledge of vaccines impact their vaccine hesitancy?

\section{Methods}

The purpose of this study is to assess the vaccine hesitancy of adolescents and the various factors which influence their vaccine resistance. Specifically, these factors include experience with the COVID-19 pandemic, whether they receive a influenza vaccine routinely, and their knowledge of vaccines. In determining whether these factors influence an adolescent's vaccine hesitancy, a greater understanding of the issue may be reached, thus leading to a clear solution.

The hypotheses for this study include:

$>$ If an adolescent knows someone who has COVID-19 or someone who has died from COVID-19 then they will have a lower vaccine hesitancy, making it more likely that they will get a vaccine for COVID-19 once it is produced.

$>$ If an adolescent commonly gets the influenza vaccine then they will have a lower vaccine hesitancy and be more likely to get the vaccine for COVID-19 once it is produced.

$>$ If an adolescent has a lower vaccine hesitancy then they will be more willing to receive a vaccine for COVID19.

$>$ If an individual has less knowledge about vaccines then they will have a higher vaccine hesitancy.

Materials:

> 101 students from the researcher's high school

$>$ Computer

$>$ Online survey 
The independent variables for this study include experience with COVID-19, routine flu vaccinations, willingness to receive a vaccination for COVID-19, and knowledge of vaccinations. The dependent variable for this study is the average vaccine hesitancy score of adolescents.

In order to obtain this information a survey composed of four sections was created. In order to see the survey in its entirety please reference the Appendix. The survey first required the signatures of the participants in addition to the signature of a parent or guardian, if the student was under the age of 18, to indicate assent and consent to participate in the research study. Following this, the survey has several demographic questions to determine details regarding participants in the sample. In this section there were also questions asked concerning experience with COVID-19 and a question that inquired about the periodicity that the student receives a flu vaccine.

After obtaining general information about participants in the sample, the next section includes 9 questions in which the possible answer choices are "incorrect, do not know, and correct" to gauge the student's knowledge of vaccines. These questions were derived from an already developed survey found in the peer-reviewed article "Measuring people's knowledge about vaccination: Developing a one-dimensional scale" by Alexandra Zingga and Michael Siegrist (2012). This knowledge survey originally had two additional questions, however, they were removed "as they reduced the scale's quality." Therefore, in order to maintain the merit of the research study, questions 10 and 11 were removed from this survey as well. Additionally, in this study it was necessary to change the wording of certain questions in order to make them easier for adolescents to understand. The third section of the survey includes the Vaccine Hesitancy Scale, a 9 question Likert Scale, to measure the participant's vaccine hesitancy. This scale was derived from the article "Measuring vaccine hesitancy: The development of a survey tool" by Heidi J. Larson et al. (2015). This particular survey has been psychometrically evaluated and its efficacy has been proven (Kempe et. al., 2020). In validating the study, Shapiro et. al removed the tenth item from the study as it was flagged "unreliable." This study removed the tenth item from the survey as well in order to maintain the quality. Furthermore, for this research study, it was necessary to modify the wording of the statements, as the scale is generally administered to parents, and the participants in this study are adolescents. In the final section of the survey there is one question with the objective of determining whether or not the participants would be willing to receive a vaccination for COVID-19. In answering no or not sure to this question, the participants are then asked to explain their reasoning.

In order to recruit individuals to participate in this study multiple science teachers at the researcher's high school were asked to administer the survey to their students. The reason the sample was gathered from science classes was because the topic of vaccine hesitancy is most related to this subject, so it was most logical to ask students from this environment. In order to determine which science classes to sample from the school a blocked cluster sample was used in which several different classes throughout the day were selected where all of the students in the class were asked to respond. The different blocks in the study are regulars science classes, honors science classes, AP science classes, co-taught science classes, and electives. The random sample was selected by the use of a random number generator in which four classes from each block were chosen. The survey was then sent to the teachers of each class and administered to the students, along with a consent and assent form attached. The purpose of asking teachers to administer the survey to students was to obtain a high rate of participation. The number of participants in the study is 101.

Due to the nature of the study, all participants are adolescents. Other inclusion criteria for the participants in the sample is that they are all students of the researcher's high school. Additionally, the sample includes students that are male or female, students in all levels of science classes, and students who chose hybrid or all-online for their school format. Exclusion criteria for the study includes students who are not enrolled in a science course at the researcher's high school. This would include students who partake in a science course at the nearby community college, which is an option offered through the high school. Moreover, there are no risks associated with the study, as all responses will be analyzed anonymously. One possible inconvenience of participating in the study would be the time it takes to complete; however, as the survey only takes about 10 to 15 minutes this is a very slight inconvenience, and it can be solved by allowing the teachers to choose a time which is most convenient for the class to participate. 


\section{Analysis}

\section{Sample Demographics}

In total there are 101 participants who completed the survey. Of these participants, $36.6 \%$ of the respondents are male and $63.4 \%$ of the respondents are female. The mean age of participants is 16.495 with a standard deviation of 1.213. The range of ages is from 13 to 18 . The median is $17.76 .2 \%$ of the participants said that they began the school year in the hybrid model and the remaining $23.8 \%$ chose to be all online at the beginning of the year. $30.693 \%$ of the participants are enrolled in an AP science class, $16.832 \%$ of the participants are enrolled in an honors science class, and $52.476 \%$ of the participants are enrolled in a regulars science class, which includes those in co-taught classes and elective classes.

Table 1. Demographics of Participants in the Study

\begin{tabular}{|l|l|}
\hline & Percent of Participants \\
\hline Male & $36.6 \%$ \\
\hline Female & $63.4 \%$ \\
\hline Hybrid School Model & $76.2 \%$ \\
\hline All-Online School Model & $23.8 \%$ \\
\hline AP Science Classes & $30.693 \%$ \\
\hline Honors Science Classes & $16.832 \%$ \\
\hline Regular Science Classes & $52.476 \%$ \\
\hline
\end{tabular}

Table 2. Age Distribution of Participants in the Study

\begin{tabular}{|l|l|l|l|l|l|}
\hline & Mean & $\begin{array}{l}\text { Standard Devi- } \\
\text { ation }\end{array}$ & Minimum & Median & Maximum \\
\hline Age & 16.495 & 1.213 & 13 & 17 & 18 \\
\hline
\end{tabular}

\section{Hypothesis 1}

$>$ If an adolescent knows someone who has COVID-19 or someone who has died from COVID-19 then they will have a lower vaccine hesitancy, making it more likely that they will get a vaccine for COVID-19 once it is produced. 
To study this hypothesis, two different questions were asked in the survey to gauge an individual's experience with COVID-19. The first question asked whether or not the individual knew someone who had COVID-19, and the second question inquired whether or not the person knew someone who had died of COVID-19.

To analyze this hypothesis two difference of means tests were performed. The factors for this hypothesiswhether or not the participant knew someone who had COVID-19 and whether or not the participant knew someone who had died of COVID-19-were compared with the average vaccine hesitancy score of each sample.

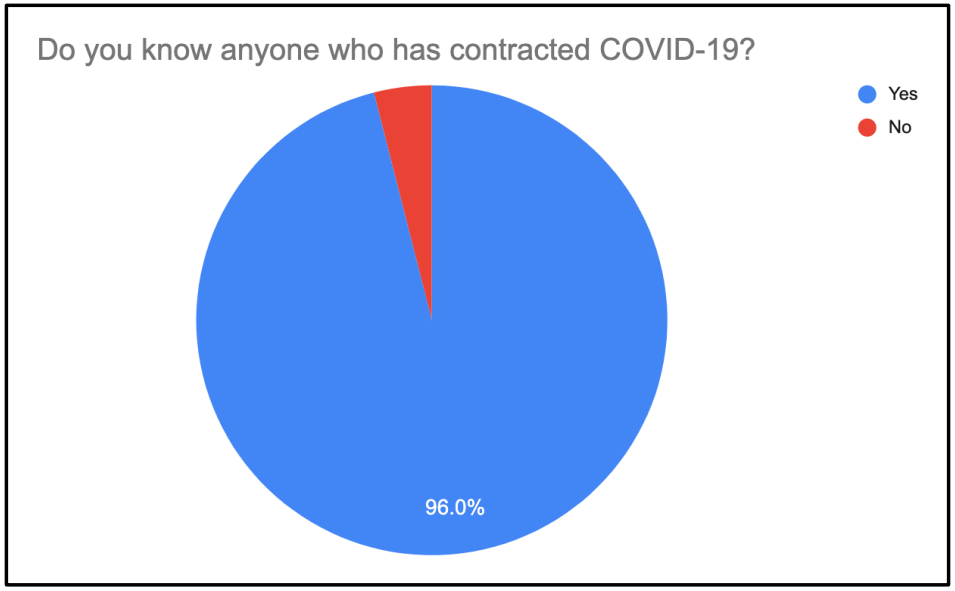

Figure 1. Participants Who Know Someone That Has Contracted COVID-19

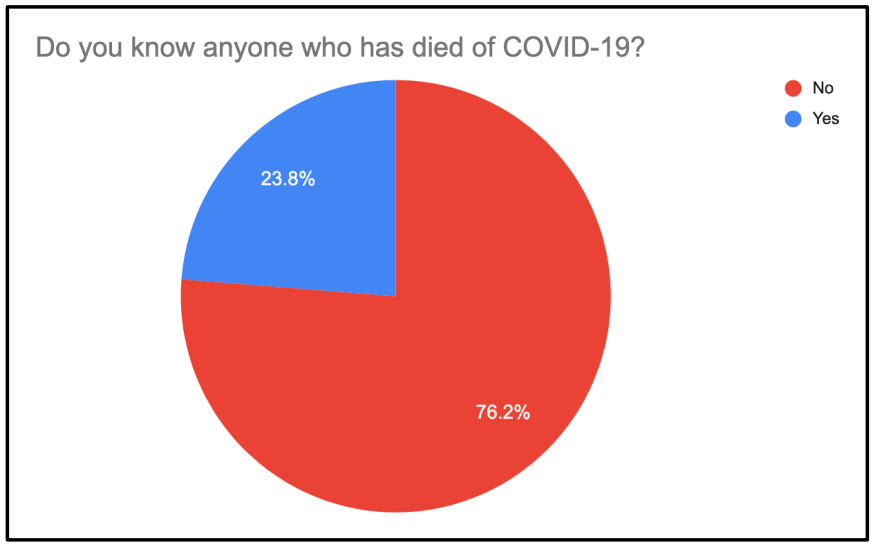

Figure 2. Participants Who Know Someone That Has Died of COVID-19

Table 3. Knows Someone Who Has Had COVID-19 Vs. Mean Vaccine Hesitancy Score

\begin{tabular}{|l|l|l|l|l|l|l|l|}
\hline & Mean Score & $\begin{array}{l}\text { Standard } \\
\text { Deviation }\end{array}$ & Minimum & Q1 & Median & Q3 & Maximum \\
\hline Knows & 18.30927835 & 5.111997377 & 9 & 14 & 18 & 21 & 34 \\
\hline
\end{tabular}




\begin{tabular}{|l|l|l|l|l|l|l|l|}
\hline Someone & & & & & & & \\
\hline $\begin{array}{l}\text { Does Not } \\
\text { Know } \\
\text { Someone }\end{array}$ & 20.25 & 11.67261753 & 10 & 12.25 & 17.5 & 25.5 & 36 \\
\hline
\end{tabular}

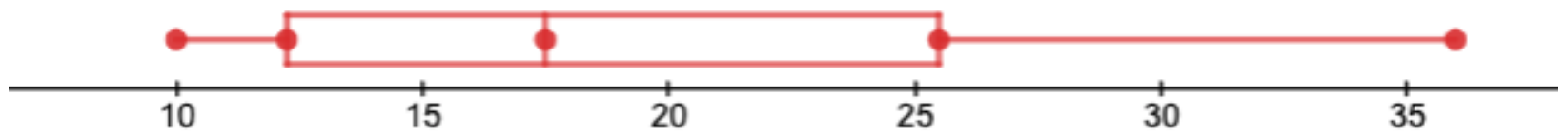

Figure 3. Distribution of Sample for Participants Who Do Not Know Someone Who Has Had COVID-19

Note: The sample size for participants who do not know someone who has had COVID-19 was only 4, which does not satisfy the conditions necessary to establish normality, allowing for a statistical test to be run. Therefore, to determine normality of the sample and satisfy the necessary conditions, this box plot was created in order to depict that the distribution of the data is normal. However, the box plot is unable to show the normality of the data, as the graph is skewed to the right.

Due to the fact that the box plot was unable to show that the sample of participants who do not know someone who has contracted COVID-19 is distributed normally, a statistical analysis of the data is unable to be conducted. Therefore, it cannot be concluded from the information collected in this study that individuals who do not know someone who has contracted COVID-19 will have an average vaccine hesitancy score that is higher than someone who does know someone who has had COVID-19.

Table 4. Knows Someone Who Has Died of COVID-19 Vs. Mean Vaccine Hesitancy Score

\begin{tabular}{|l|c|c|c|c|c|c|c|}
\hline & Mean Score & $\begin{array}{c}\text { Standard } \\
\text { Deviation }\end{array}$ & Minimum & Q1 & Median & Q3 & Maximum \\
\hline $\begin{array}{l}\text { Knows } \\
\text { Someone } \\
\text { COVID }\end{array}$ & 17.58333333 & 4.754098156 & 10 & 14 & 17 & 20.5 & 27 \\
\hline $\begin{array}{l}\text { Does Not } \\
\text { Know } \\
\text { Someone }\end{array}$ & 18.63636364 & 5.609927973 & 9 & 14 & 18 & 21 & 36 \\
\hline
\end{tabular}

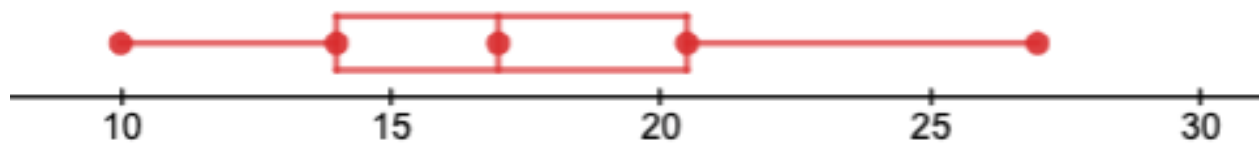

Figure 4. Distribution of Sample for Participants Who Know Someone Who Has Died of COVID-19 
Note: The sample size for participants who know someone who has died of COVID-19 was only 24, which does not satisfy the conditions necessary to establish normality, allowing for a statistical test to be run. Therefore, to determine normality of the sample and satisfy the necessary conditions, this box plot was created that depicts the data as normally distributed.

Performing a difference of means test for the average vaccine hesitancy score of individuals who know someone who has died from COVID-19 and individuals who do not know someone who has died from COVID-19, the sample of participants who do not know someone was defined as $\mu_{1}(n=77)$, and the sample of participants who do know someone was defined as $\mu_{2}(n=24)$. The null hypothesis states that $\mu_{1}=\mu_{2}$, and the alternative hypothesis states that $\mu_{1}>\mu_{2}$. The mean vaccine hesitancy score for the first sample was $18.636(\mathrm{~s}=5.610)$, while the mean vaccine hesitancy score for the second sample was $17.583(\mathrm{~s}=4.754)$. The $\mathrm{p}$-value resulting from this difference of means test was 0.187 ; therefore, the data fails to reject the null hypothesis $(\alpha=0.05)$.

\section{Hypothesis 2}

$>$ If an adolescent commonly gets the influenza vaccine then they will have a lower vaccine hesitancy and be more likely to get the vaccine for COVID-19 once it is produced.

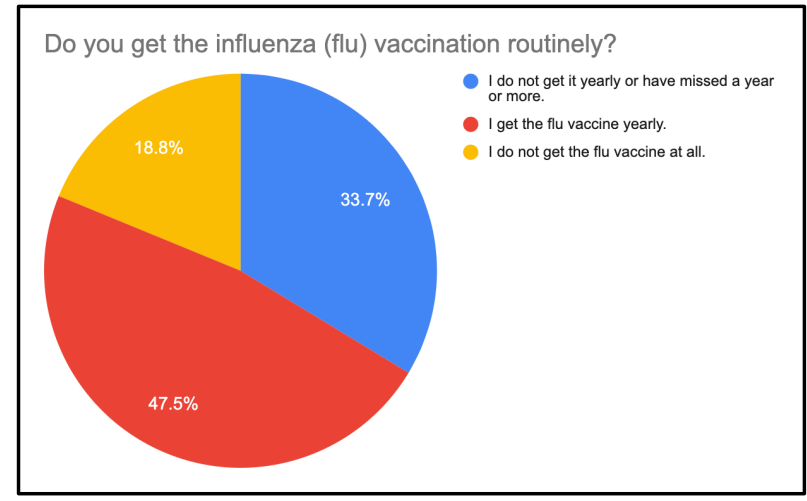

Figure 5. Frequency That Participants Receive Influenza Vaccinations

Table 5. Flu Vaccine Vs. Mean Vaccine Hesitancy Score

\begin{tabular}{|l|c|c|c|c|c|c|c|}
\hline & Mean Score & $\begin{array}{c}\text { Standard } \\
\text { Deviation }\end{array}$ & Minimum & Q1 & Median & Q3 & Maximum \\
\hline $\begin{array}{l}\text { Gets the Flu } \\
\text { Vaccine } \\
\text { Routinely }\end{array}$ & 17.27083333 & 4.992503245 & 10 & 13.75 & 17 & 19.25 & 34 \\
\hline
\end{tabular}




\begin{tabular}{|l|l|l|l|l|l|l|l|}
\hline $\begin{array}{l}\text { Does Not } \\
\text { Get the Flu } \\
\text { Vaccine } \\
\text { Routinely }\end{array}$ & 19.39622642 & 5.627335832 & 9 & 15 & 19 & 22 & 36 \\
\hline
\end{tabular}

To study this hypothesis, the survey asked the question: "Do you get the influenza (flu) vaccination routinely?" The possible answer choices were "I get the flu vaccine yearly, I do not get it yearly or have missed a year or more, and I do not get the flu vaccine at all." The participants were then split into two samples based on their responses. Those who indicated that they do not get the vaccine routinely or at all are defined as $\mu_{1}(\mathrm{n}=53)$, and participants who indicated that they get the vaccine yearly were defined as $\mu_{2}(n=48)$. A difference of means T-test was performed in which the null hypothesis states that $\mu_{1}=\mu_{2}$, and the alternative hypothesis states that $\mu_{1}>\mu_{2}$. The mean vaccine hesitancy score for the first sample was 19.396 (s=5.627) and the mean vaccine hesitancy score for the second sample was 17.271 (4.995). The resulting $p$-value of the test was 0.025 , thus the null hypothesis was rejected $(\alpha=0.05)$. Therefore, it may be concluded with $95 \%$ confidence that if an individual does not receive a routine influenza vaccination then their average vaccine hesitancy score will be higher than an individual who does receive a routine influenza vaccination.

\section{Hypothesis 3}

$>$ If an adolescent has a lower vaccine hesitancy then they will be more willing to receive a vaccine for COVID-19.

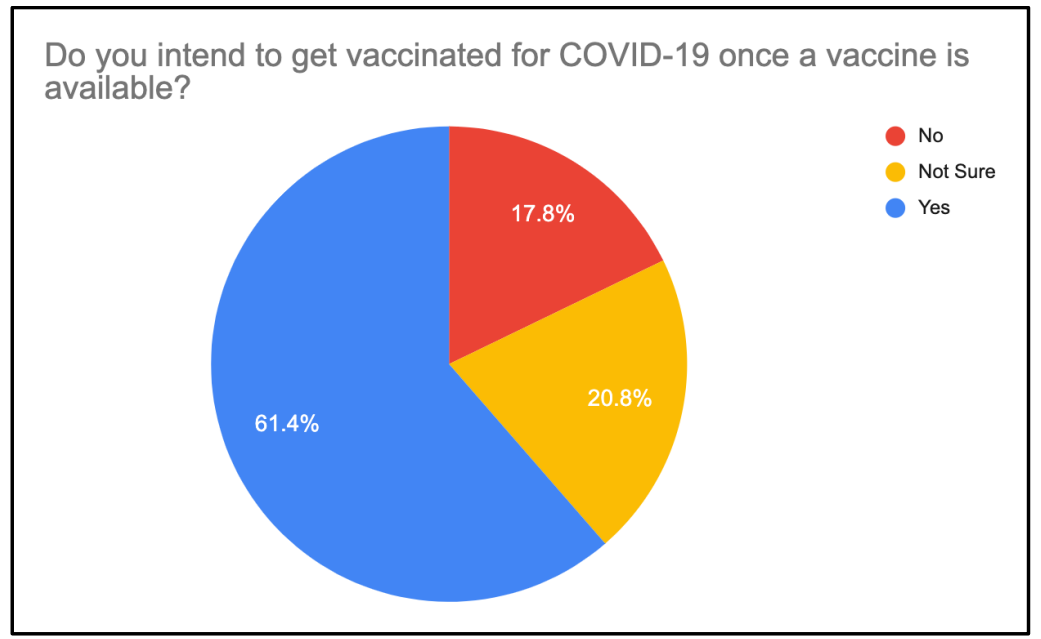

Figure 6. Intent to Get Vaccinated for COVID-19 Once a Vaccine is Available

Table 6. Willingness to Get the COVID-19 Vaccine Vs. Mean Vaccine Hesitancy Score

\begin{tabular}{|l|c|c|c|c|c|c|c|}
\hline & Mean Score & $\begin{array}{l}\text { Standard } \\
\text { Deviation }\end{array}$ & Minimum & Q1 & Median & Q3 & Maximum \\
\hline $\begin{array}{l}\text { Willing to } \\
\text { Get } \\
\text { VaviD } \\
\text { Vaccine }\end{array}$ & 15.46774194 & 3.103238615 & 9 & 13 & 15 & 17 & 22 \\
\hline
\end{tabular}




\begin{tabular}{|l|l|l|l|l|l|l|l|}
\hline $\begin{array}{l}\text { Not Will- } \\
\text { ing to Get } \\
\text { COVID } \\
\text { Vaccine }\end{array}$ & 23.02564103 & 5.070488295 & 13 & 20 & 23 & 26 & 36 \\
\hline
\end{tabular}

A difference of means test was performed to determine whether the average of the vaccine hesitancy score would increase or decrease in accordance with a participant's willingness to receive the COVID-19 vaccine. The sample of participants who responded "no" or "not sure" are defined by $\mu_{1}(n=39)$, and the sample of participants who responded "yes" are defined by $\mu_{2}(\mathrm{n}=62)$. The null hypothesis states that $\mu_{1}=\mu_{2}$, and the alternative hypothesis was established that $\mu_{1}>\mu_{2}$. The mean vaccine hesitancy score of participants who are not willing to get a COVID-19 vaccination was 23.026 ( $\mathrm{s}=5.07)$, and the mean vaccine hesitancy score of participants who are willing to get a COVID-19 vaccination was 15.468 ( $\mathrm{s}=3.103$ ). The resulting $\mathrm{p}$-value of the difference of means T-test was $1.85 \times 10^{-}$ ${ }^{10}$. Therefore, this study rejects the null hypothesis $(\alpha=0.01)$ indicating with greater than $99 \%$ confidence that the average vaccine hesitancy score of individuals who are not willing to get the COVID-19 vaccination are higher than the average vaccine hesitancy score for an individual who is willing to get the COVID-19 vaccination.

\section{Hypothesis 4}

If an individual has less knowledge about vaccines then they will have a higher vaccine hesitancy.

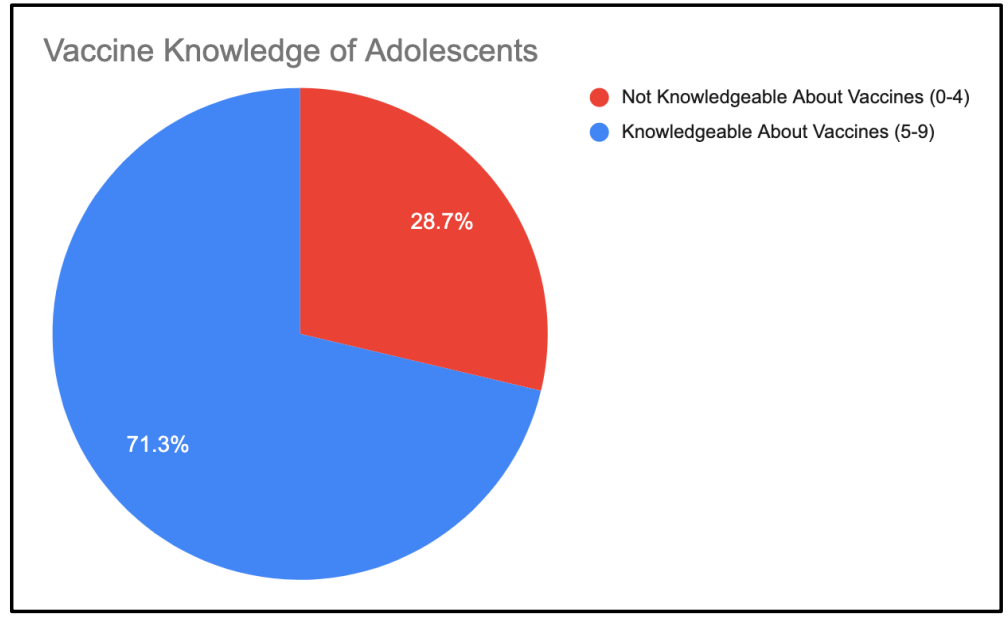

Figure 7. Vaccine Knowledge of Adolescents

Table 7. Knowledge of Vaccines Vs. Mean Vaccine Hesitancy Score

\begin{tabular}{|l|c|c|c|c|c|c|c|}
\hline & Mean Score & $\begin{array}{c}\text { Standard } \\
\text { Deviation }\end{array}$ & Minimum & Q1 & Median & Q3 & Maximum \\
\hline $\begin{array}{l}\text { Knowl- } \\
\text { edgeable } \\
\begin{array}{l}\text { About } \\
\text { Vaccines }\end{array}\end{array}$ & 16.68055556 & 4.337281517 & 9 & 14 & 16 & 19.25 & 32 \\
\hline
\end{tabular}




\begin{tabular}{|l|l|l|l|l|l|l|l|}
\hline $\mathbf{( 5 - 9 )}$ & & & & & & & \\
\hline $\begin{array}{l}\text { Not } \\
\text { Knowl- } \\
\text { edgeable } \\
\begin{array}{l}\text { About } \\
\text { Vaccines } \\
(\mathbf{0 - 4})\end{array}\end{array}$ & 22.62068966 & 5.564002883 & 10 & 19 & 22 & 27 & 36 \\
\hline
\end{tabular}

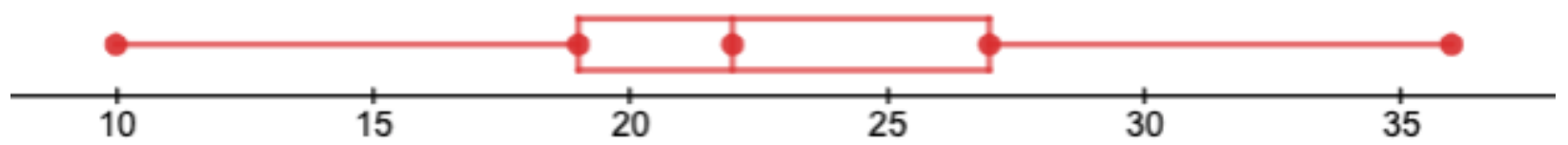

Figure 8. Distribution of Sample for Participants Who Are Not Knowledgeable About Vaccines

Note: The sample size for participants who are not knowledgeable about vaccines was only 29 , which does not satisfy the conditions necessary to establish normality, allowing for a statistical test to be run. Therefore, to determine normality of the sample and satisfy the necessary conditions, this box plot was created that depicts the data as normally distributed.

For the final hypothesis, a difference of means test was completed in order to determine that an individual who is more knowledgeable about vaccines will have a lower vaccine hesitancy score. The peer-reviewed article where the Vaccine Knowledge Survey was obtained from, "Measuring people's knowledge about vaccination: Developing a one-dimensional scale," did not delineate what score qualifies an individual as "knowledgeable" about vaccinations, so in order to categorize the sample into "knowledgeable participants" and "not as knowledgeable participants" in the most equitable way the first half of the scale-scores 0 to 4-were considered not knowledgeable, and the second half of the scale-scores 5 to 9-were considered knowledgeable.

The sample of participants who are not knowledgeable about vaccines is defined by $\mu_{1}(\mathrm{n}=29)$, and the sample of participants who are knowledgeable about vaccines is defined by $\mu_{2}(\mathrm{n}=72)$. The null hypothesis states that $\mu_{1}=\mu_{2}$, and the alternative hypothesis states that $\mu_{1}>\mu_{2}$. The mean vaccine hesitancy score of individuals who are less knowledgeable about vaccines is 22.621 ( $\mathrm{s}=5.564)$, and the mean vaccine hesitancy score of individuals who are more knowledgeable about vaccines is $16.681(\mathrm{~s}=4.337)$. The difference of means T-test determined a $\mathrm{p}$-value of $9.126 \times 10^{-}$ ${ }^{10}$; therefore, the null hypothesis is rejected $(\alpha=0.01)$, and it is concluded with greater than $99 \%$ confidence that the average vaccine hesitancy score of an individual who is less knowledgeable about vaccines will be greater than the average vaccine hesitancy score of an individual who is more knowledgeable about vaccines. 


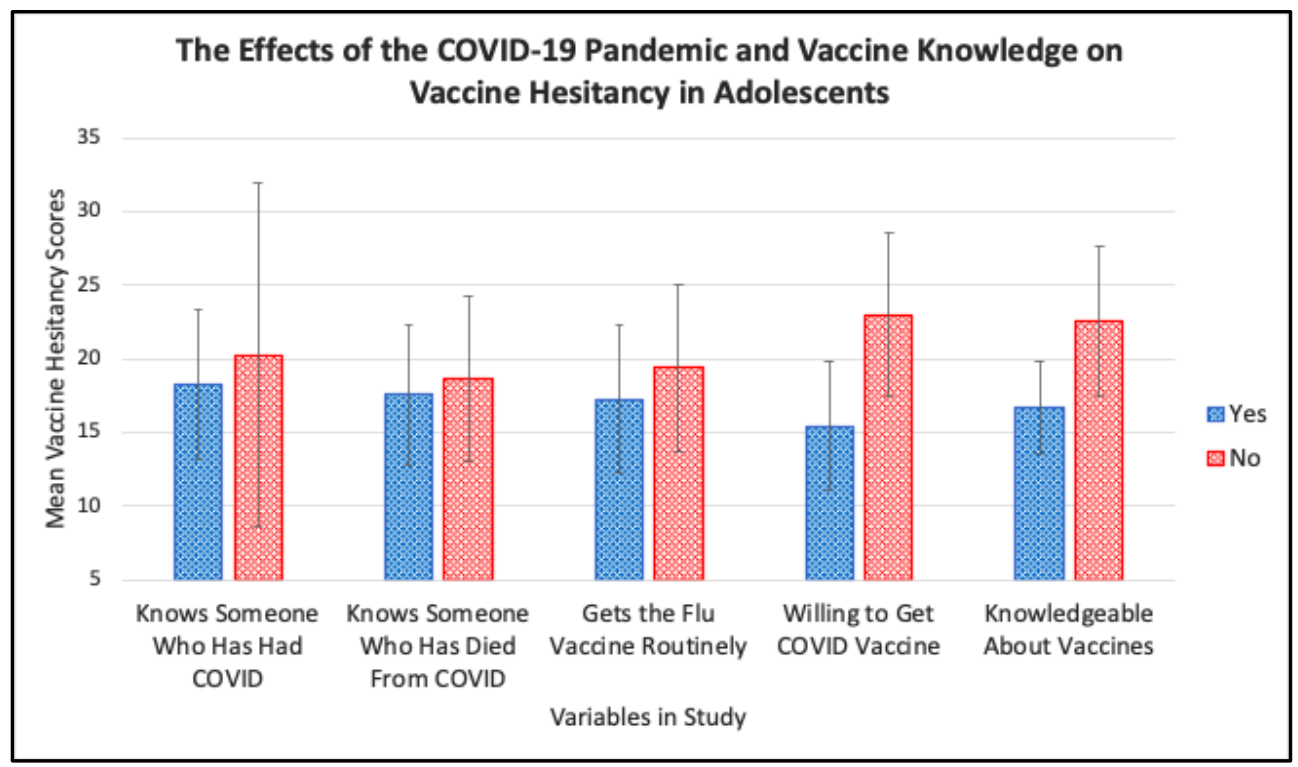

Figure 9. The Effects of the COVID-19 Pandemic and Vaccine Knowledge on Vaccine Hesitancy in Adolescents

This chart depicts the average vaccine hesitancy scores as compared to the various hypotheses explored in the study. The first two variables listed represent the first hypothesis which explored the effects of knowing someone that has contracted COVID-19 or knowing someone that has died of COVID-19 on an individual's vaccine hesitancy. The standard deviation bars completely overlap for these two variables showing that the results of these comparisons were not statistically significant. The standard deviation bars for the next variable, which compared the effects of receiving a routine influenza vaccination on the vaccine hesitancy scores, do not completely overlap, thus the results for this analysis are statistically significant. For the next variable comparing the effects of being willing to receive a COVID-19 vaccination on the vaccine hesitancy scores there is a large area in which the standard deviation bars do not overlap, which shows that the results for this comparison are statistically significant. This is also shown with the last variable comparing the knowledge of vaccines to the average vaccine hesitancy scores.

\section{Additional Analysis}

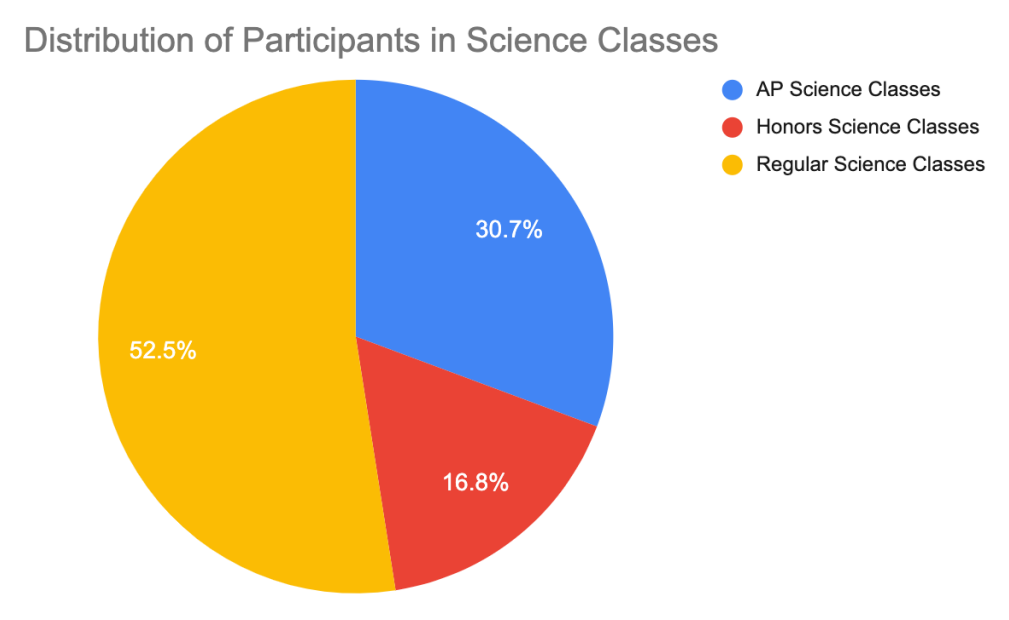

Figure 10. Distribution of Participants in Science Classes

Table 8. Science Class Vs. Knowledge of Vaccines Scores 


\begin{tabular}{|l|c|c|c|c|c|c|c|}
\hline & $\begin{array}{c}\text { Mean } \\
\text { Knowledge } \\
\text { Score }\end{array}$ & $\begin{array}{c}\text { Standard } \\
\text { Deviation }\end{array}$ & Minimum & Q1 & Median & Q3 & Maximum \\
\hline $\begin{array}{l}\text { AP or Hon- } \\
\text { ors Science } \\
\text { Class }\end{array}$ & 6.4583 & 1.9347 & 2 & 5 & 6 & 8 & 9 \\
\hline $\begin{array}{l}\text { Regular } \\
\text { Science } \\
\text { Class }\end{array}$ & 4.3396 & 2.5865 & 0 & 2 & 5 & 6 & 9 \\
\hline
\end{tabular}

In addition to analyzing the data collected to either accept or reject the hypotheses of this study, additional analysis was also completed to explore various other factors from the survey. For example, a difference of means test was performed to determine if an individual in an AP or honors science course would have a greater knowledge of vaccines. A difference of means test was completed in which the sample of participants in honors and AP science classes was defined as $\mu_{1}(n=48)$ and the sample of participants in regular science classes was defined as $\mu_{2}(n=53)$. The null hypothesis states that $\mu_{1}=\mu_{2}$, and the alternative hypothesis states that $\mu_{1}>\mu_{2}$. The average knowledge of vaccines scores on a scale from 1 to 9 for the first sample was 6.458 ( $(\mathrm{s}=1.935)$, and the average knowledge of vaccines scores for the second sample was $4.340(\mathrm{~s}=2.586)$. The resulting $\mathrm{p}$-value was $1.196 \times 10^{-5}$, therefore the null hypothesis is rejected $(\alpha=0.01)$ and it can be concluded with greater than $99 \%$ confidence that if an individual is in an AP or honors science class they will be more knowledgeable about vaccinations than an individual who is in a regular science class.

While the study was unable to confirm the hypothesis that an individual who knows someone that has died of COVID-19 will be less vaccine hesitant, an additional analysis was completed to determine whether or not an individual who knows someone that has died of COVID-19 will be more willing to receive a vaccination for COVID19. To determine this, a difference of proportions test was performed where the proportion of participants who know someone that has died of COVID-19 was defined as $\mathrm{P}_{1}(\mathrm{n}=24)$ and the proportion of individuals who do not know someone that has died of COVID was defined as $\mathrm{P}_{2}(\mathrm{n}=78)$. The proportion of participants willing to get a vaccination for COVID-19 in the first sample is $66.67 \%$ and the proportion of participants willing to get a vaccination for COVID19 in the second sample is $58.97 \%$. The resulting p-value of the difference of proportions test was 0.2696 ; therefore, the study fails to reflect the null hypothesis $(\alpha=0.05$ ), and it cannot be concluded that individuals who know someone that has died of COVID-19 will be more willing to receive a vaccination than individuals who do not know someone that has died of COVID-19.

Table 9. Distribution of Responses to Vaccine Hesitancy Survey 


\begin{tabular}{|c|c|c|c|c|c|}
\hline & Strongly Disagree & Disagree & $\begin{array}{l}\text { Neither Agree } \\
\text { Nor Disagree }\end{array}$ & Agree & $\begin{array}{c}\text { Strongly } \\
\text { Agree }\end{array}$ \\
\hline $\begin{array}{l}\text { Vaccines are important } \\
\text { for my health. }\end{array}$ & 2 & 4 & 6 & 37 & 52 \\
\hline $\begin{array}{l}\text { Getting vaccines is a } \\
\text { good way to protect } \\
\text { myself from disease. }\end{array}$ & 0 & 2 & 7 & 38 & 54 \\
\hline Vaccines are effective. & 0 & 1 & 12 & 44 & 44 \\
\hline $\begin{array}{l}\text { Receiving vaccinations } \\
\text { is important for the } \\
\text { health of others in } \mathrm{my} \\
\text { community. }\end{array}$ & 0 & 4 & 9 & 28 & 60 \\
\hline $\begin{array}{l}\text { All vaccines offered by } \\
\text { the government pro- } \\
\text { gram in my community } \\
\text { are beneficial. }\end{array}$ & 2 & 12 & 35 & 32 & 20 \\
\hline $\begin{array}{l}\text { The information I re- } \\
\text { ceive about vaccines } \\
\text { from the vaccine pro- } \\
\text { gram is reliable and } \\
\text { trustworthy. }\end{array}$ & 1 & 3 & 34 & 44 & 19 \\
\hline $\begin{array}{l}\text { Generally I do what my } \\
\text { doctor or health care } \\
\text { provider recommends } \\
\text { about vaccines. }\end{array}$ & 0 & 3 & 7 & 38 & 53 \\
\hline $\begin{array}{l}\text { New vaccines carry } \\
\text { more risk than older } \\
\text { vaccines. }\end{array}$ & 10 & 25 & 30 & 23 & 13 \\
\hline $\begin{array}{l}\text { I am concerned about } \\
\text { serious adverse effects } \\
\text { of vaccines. }\end{array}$ & 20 & 28 & 30 & 20 & 3 \\
\hline
\end{tabular}

\section{Conclusion}


The results of the multiple difference of means T-tests were able to substantiate three of the four hypotheses of this study. The analysis shows that an adolescent who recieves an influenza vaccination routinely will have an average vaccine hesitancy score that is lower than adolescents who do not receive the flu vaccine routinely $(\alpha=0.05)$. Additionally, adolescents who are willing to receive a vaccination for COVID-19 once it is available have an average vaccine hesitancy score that is lower than adolescents who are not willing to receive a vaccination for COVID-19 $(\alpha=0.01)$. The analysis also exemplifies that adolescents who are more knowledgeable about vaccines will have a lower vaccine hesitancy score than those who are less knowledgeable about vaccines $(\alpha=0.01)$. The data collected in this study was not able to substantiate the claim that individuals who know someone who has died from COVID-19 or know someone who has contracted COVID-19 will be less vaccine hesitant.

As of January 31, 2021 the number of cases of COVID-19 in the United States according to the "COVID Data Tracker" published by the Centers For Disease Control and Prevention was 25,921,703. This data shows COVID19 is ubiquitous, and it should be expected that the majority of people know someone who has contracted COVID-19 at this point in the pandemic. Therefore, a limitation to the study is that not enough individuals who do not know someone that has contracted COVID-19 was able to be established. This resulted in a skewed distribution that was not able to be analyzed. Additionally, the sample of individuals who knew someone that had died of COVID-19 was only 24. Had this sample of individuals been larger it may have been possible to substantiate the claim that individuals who know someone that has died of COVID-19 will be less vaccine hesitant. In future explorations of this study, it is recommended that a greater number of participants is obtained. Furthermore, the study was not able to show that the pandemic had an effect on individuals' vaccine hesitancy. This may be partially due to the fact that there were not enough specific questions regarding experience of the pandemic. If more specific questions were asked to gauge an individual's experience, it would have provided for a more clear analysis of how this pandemic has impacted the vaccine hesitancy of individuals. In further exploration of this topic, it is recommended that a more detailed section with questions related to COVID-19 experience is added for a depthful analysis and comparison.

In addition to the quantitative data that was collected from the survey, following the question regarding the participant's willingness to receive the COVID-19 vaccination, the students who responded no or not sure were asked to explain why they felt this way. This information in particular may be useful in proposing a solution to lower vaccine hesitancy in adolescents. Many of the reasons proposed by the participants are established due to similar beliefs. For example, the majority of responses appertained to the participants' fear of the long term effects of the COVID-19 vaccine, the fact that many of them are not as susceptible to the adverse effects of COVID-19, and their parent's hesitancy towards the vaccination. The quantitative data obtained in the study was able to determine with great statistical significance that individuals who are more vaccine hesitant will be less willing to receive a vaccine for COVID19, and this qualitative data which was acquired explains the reasoning behind their resistance. Furthermore, the additional analysis of the responses for the Vaccine Hesitancy Scale showed that $35.64 \%$ of participants were concerned that new vaccines carry more risk than older vaccines. This is the most significant reason, according to the study, that adolescents are hesitant to receive vaccinations. Therefore, the recommendations going forward would be to educate the public, specifically adolescents, on why they should not be as hesitant towards the COVID-19 vaccine in particular, especially in the midst of a grievous pandemic.

Misinformation can be easily spread due to technology, which results in individuals not being as knowledgeable about certain issues. For example, as this study shows, adolescents are wary of the COVID-19 vaccine due to the rapid production and skepticism of unknown long-term effects. However, the Mayo Clinic published an article titled "COVID-19 vaccine myths debunked" in which they explain that even though the vaccine was developed rapidly, this does not mean that safety precautions and protocols were bypassed. Additionally, the article ensures that the clinic will only recommend the use of vaccines that they are sure are safe. Not only this, but the article "COVID-19 Vaccines: Myth Versus Fact" published by John Hopkins explains that the methods used by Pfizer/BioNTech and Moderna to create the vaccine have been in development for years. Moreover, studies have determined that both the Pfizer/BioNTech and Moderna vaccines are about 95\% effective and have no serious or life-threatening side effects. The shortterm side effects that accompany any vaccination including headaches, chills, fatigue, and muscle pain are just an 
indication that the "immune system is responding to the vaccine" (COVID-19 vaccine myths debunked). In order to emerge from this pandemic it is necessary to gain herd immunity for COVID-19, which will not be possible unless the vaccine hesitancy of all individuals is greatly reduced so that more people are willing to get vaccinated against the virus.

The implications of this study are especially important now as it is crucial to lower the vaccine hesitancy of individuals in order to vaccinate against COVID-19 and increase herd immunity to end the pandemic. Not only is it necessary to regulate the use of the Vaccine Hesitancy Scale to gauge the vaccine hesitancy of the population, but the universal use of one specific scale rather than many different scales will make it easier to analyze and compare data as well. In gathering more information about the vaccine hesitancy of the population it is easier to specify what needs to be done to make individuals less resistant to vaccines. This study indicates that in order to make adolescents less vaccine hesitant it is important to educate them about vaccinations to increase their knowledge. Increasing an adolescent's knowledge of vaccines will make them less hesitant and in turn, more willing to receive a vaccination for COVID-19 once one is available to them. To educate youth on vaccinations, states could mandate the discussion of vaccines in the curriculum of science classes. Additionally, ad campaigns on social media could potentially be used to make adolescents more aware of the issue of vaccine hesitancy and the dangers it poses to public health. Not only is advocating for greater vaccine knowledge important, but opposing misinformation in the age of technology is also crucial. It is incredibly necessary to take globalized action against this dangerous issue of vaccine hesitancy due to the great risk that it imposes on the health and safety of the public. The efficacy of vaccines has been proven time and time again, and their necessity is especially overwhelming now, thus making it increasingly clear how crucial it is to take steps to combat the growing problem.

\section{Acknowledgments}

I would like to thank my advisor Ms. Lori Cohen for helping me with this project.

\section{References}

Barello, S., Nania, T., Dellafiore, F., Graffigna, G., \& Caruso, R. (2020). 'Vaccine hesitancy' among university students in Italy during the COVID-19 pandemic. European Journal of Epidemiology, (35), 781-783. https://doi.org/10.1007/s10654-020-00670-z

COVID-19 vaccine myths debunked. (2020, December 8). Mayo Clinic Health System. https://www.mayoclinichealthsystem.org/hometown-health/featured-topic/ covid-19-vaccine-myths-debunked

COVID-19 vaccines: Myth versus fact. (2021, January 13). John Hopkins Medicine. https://www.hopkinsmedicine.org/health/conditions-and-diseases/coronavirus/ covid-19-vaccines-myth-versus-fact

COVID data tracker. (n.d.). Centers for Disease Control and Prevention.

Retrieved January 31, 2021, from https://covid.cdc.gov/covid-data-tracker/ \#cases_totalcases

Hussain, A., Ali, S., Ahmed, M., \& Hussain, S. (2018). The Anti-vaccination Movement: A Regression in Modern Medicine. Cureus, 10(7), e2919. https://doi.org/10.7759/cureus.2919 
Isaacs D. (2019). Vaccine hesitancy and anti-vaccination movements. Journal of paediatrics and child health, 55(11), 1293-1294. https://doi.org/10.1111/jpc.14581

Kempe, A., Saville, A. W., Albertin, C., Zimet, G., Breck, A., Helmkamp, L., Vangala, S., Dickinson, L. M., Rand, C., Humiston, S., \& Szilagyi, P. G. (2020). Parental hesitancy about routine childhood and influenza vaccinations: A national survey. Pediatrics, (146), 1-12. https://doi.org/10.1542/peds.2019-3852

Larson, H. J., Jarrett, C., Schulz, W. S., Chaudhuri, M., Zhou, Y., Dube, E., Schuster, M., MacDonald, N. E., Wilson, R., \& The SAGE Working Group on Vaccine Hesitancy. (2015). Measuring vaccine hesitancy: The development of a survey tool. Vaccine, (33), 4165-4175. https://doi.org/10.1016/j.vaccine.2015.04.037

Leask J. (2011). Target the fence-sitters. Nature, 473(7348), 443-445. https://doi.org/10.1038/473443a

Maltezou, H. C., Theodoridou, K., \& Poland, G. (2020). Influenza immunization and COVID-19. Vaccine, (38), 6078-6079. https://doi.org/10.1016/j.vaccine.2020.07.058

McAteer, J., Yildirim, I., \& Chahroudi, A. (2020). The VACCINES Act: Deciphering Vaccine Hesitancy in the Time of COVID-19. Clinical infectious diseases : an official publication of the Infectious Diseases Society of America, 71(15), 703-705. https://doi.org/10.1093/cid/ciaa433

Noor, I. (2020, June 10). Confirmation bias. SimplyPsychology. https://www.simplypsychology.org/confirmationbias.html?scrlybrkr=349e998a

Palamenghi, L., Barello, S., Boccia, S., \& Grafgna, G. (2020). Mistrust in biomedical research and vaccine hesitancy: the forefront challenge in the battle against COVID-19 in Italy. European Journal of Epidemiology, (35), 785-788. https://doi.org/10.1007/s10654-020-00675-8

Sanche, S., Lin, Y. T., Xu, C., Romero-Severson, E., Hengartner, N., \& Ke, R. (2020). High contagiousness and rapid spread of severe acute respiratory syndrome coronavirus 2. Emerging Infectious Diseases, 26(7), 1470-1477. https://doi.org/10.3201/eid2607.200282

Shapiro, G. K., Tatar, O., Dube, E., Amsel, R., Knauper, B., Naz, A., Perez, S., \& Rosberger, Z. (2017). The vaccine hesitancy scale: Psychometric properties and validation. Vaccine, (36), 660-667. https://doi.org/10.1016/j.vaccine.2017.12.043

Six common misconceptions about immunization. (n.d.). https://www.who.int/vaccine_safety/initiative/detection/immunization_misconceptions/en/

Zingga, A., \& Siegrist, M. (2012). Measuring people's knowledge about vaccination: Developing a one-dimensional scale. Vaccine, (30), 3771-3777. https://doi.org/10.1016/j.vaccine.2012.03.014 